\title{
Book Review of "Stochastic Physics and Climate Modelling" - a trillion dollar challenge
}

\author{
D. Schertzer \\ Universitée Paris-Est, Ecole des Ponts ParisTech, LEESU, Marne-la-Vallée, France
}

STOCHASTIC PHYSICS AND CLIMATE MODELLING, EDITED BY: T. PALMER AND P. WILLIAMS, CAMBRIDGE UNIVERSITY PRESS, CAMBRIDGE, UK, 480 PAGES, ISBN 978-0-521-76105-5, HARDCOVER, 2010.

The readers of Nonlinear Processes in Geophysics are well aware that the solutions to nonlinear deterministic-like equations governing weather evolution are most probably chaotic in space and time: a small scale truncation can in a finite time generate large-scale errors. This behaviour has been conjectured precisely, for the prototypical Navier-Stokes equations and is subject to a million-dollar Clay Mathematics Millennium prize. Without awaiting this mathematical conclusion, statistical theories of turbulence and corresponding stochastic models are already in constant use in a wide range of fluid mechanics applications.

The book "Stochastic Physics and Climate Modelling" edited by Palmer and Williams (2010) pushes forward these ideas in an original manner to the even more challenging and wider theme of climate change, which has an estimated worth of one trillion dollars (Stern, 2006), as recalled by the editors in their breathtaking preface. This book indeed promotes the use of stochastic, or random, processes to understand, model and predict our climate system, and in particular to resolve the presently considerable uncertainty in global and regional climate predictions.

This book corresponds to the careful updating and enlarging (seven new chapters have been added to the original eleven ones) of a thematic issue of the Royal Society introduced by Palmer and Williams (2008). This enables the book to cover a wide range of topics: low dimensional stochastic models and model reduction procedures; stochastic modelling of turbulence, diffusion and climate variability; stochastic differential equations driven by noise and their predictability; random dynamical systems and stochastic bifurcations; multiscale processes; stochastic parametrizations.

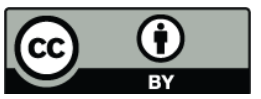

Correspondence to: D. Schertzer (daniel.schertzer@enpc.fr)
Some topics are rather mathematically oriented, others are treated at different levels including those with an emphasis on practical applications. This is particularly the case for stochastic parametrizations with the question of appropriate backscatter schemes to model the small scale feedback on larger scales.

This book also benefits from the diversity of leading experts (mathematicians, physicists, oceanographers and meteorologists) who present different facets and approaches to the book's theme. Although it has the usual drawback that navigating within a multiple authored book is more difficult than within a single authored book. There are some natural overlaps on stochastic climate modelling with the book edited by Imkeller (2001), but the latter is more mathematically oriented and focused on random dynamical systems to a revisit of Hasselmann's program, whereas this book is focused on improving climate sub-grid modelling with the help of stochastic processes. There are much fewer overlaps with the books authored by Dobrovolski (2009) and Ditlevsen (PhD thesis, 2004), both of them being more focused on time-series analysis with the help of turbulence and scaling ideas. As would be expected due to my personal biases, I have some regrets about it. I feel that these ideas are not used enough in the present book to both characterize and model the climate variability over a wide range of scale, whereas the editors do acknowledge in their introduction the legacy of Richardson and Kolmogorov on the fundamental questions of fluctuations in nonlinear processes.

In conclusion, the book "Stochastic Physics and Climate Modelling" is a timely thought-provoking book on one of the most challenging and paradoxical scientific issues: stochastic physics may well be the key to substantial progress being made in climate change modelling and prediction, and to resolve the large uncertainties that exist. It is therefore a must for anyone having a keen interest in climate modelling, especially graduate students and researchers involved in climate studies.

Published by Copernicus Publications on behalf of the European Geosciences Union and the American Geophysical Union. 


\section{References}

Ditlevsen, P. D.: Turbulence and Climate Dynamics, Copenhagen, J. \& R. Frydenberg A/S, 2004.

Dobrovolski, S. G.: Stochastic Climate Theory: Models and Applications, Berlin-Heidelberg, Springer, 2009.

Imkeller, P. and von Storch, J.-S. (Eds.): Stochastic Climate Models. Progress in Probability, Basel, Birkhäuser Verlag, 2001.
Palmer, T. and Williams P.: Introduction. Stochastic physics and climate modelling, Phil. Trans. R. Soc. A, 366(1875), 2419-2425, 2008.

Palmer, T. and Williams, P. (Eds.): Stochastic Physics and Climate Modelling, Cambridge University Press, Cambridge, UK, 2010.

Stern, N.: The Economics of Climate Change: the Stern Review, Cambridge Universisty Press, Cambridge UK, 2006. 